from this channel. If now we look upon this channel as breaking up into a capillary network-the capillaries of which are the spaces between connective-tissue or fat cells, and suppose that it acts as an irritant, as in a healing wound, the development of a lymphatic gland, with its ly mph follicles, its delicate reticulum, its slowly proliferating endothelial cells and its accumulation of lymphoid cells is comparatively easily explained. The enlargement of this gland under increased irritation, the enormous proliferation of both kinds of cells in such conditions as typhoid or septic irritation, and the like, and the actual suppuration that occurs when these processes are very intense are readily understood. The more we think over the matter the more are we struck by the similarity of the processes abovementioned, and the more does it appear necessary that the development of the lymphatic gland should be studied in order that its importance in the economy in the localisation of disease may be appreciated. The time has now gone by for connective tissues to be looked upon merely as padding and support, or even as tissue in which fat may be accumu. lated and leucocytes formed. These connective- bissue cells along with the leucocytes play a far more important rôle. Various metabolic products have been found in them, and the liver is gradually being ousted from its high place as the great metaboliser of the body, and its place is being taken, in part at any rate, by the connective-tissue cells and leucocytes; whilst it is further acknowledged that even where the liver is allowed to retain any metabolic function it is of ten merely to complete work that has already been commenced by the tissues of which we have spoken, and that so farits fanctions are excretory rather than secretory. In making such statements as these one must be extremely guarded lest functions of which hitherto we have had too exalted an opinion should now have too little importance attached to them. At present, however, there is little enough danger of the mesoblastic tissues being promoted to too prominent a position, especially if it is to be at the expense of the liver. Certainly the connective-tissue cells and leucocytes have much more in common with those of the liver than is often admitted; whilst the greater the degeneration of liver cells in any disease, the nearer in most cases does it approximate in its functions the active connective tissue cells. In both kinds of cell we find that fat may be stored up, such fat apparently in most cases being derived from the splitting up of proteids or other substances containing the fat molecule that are brought from without. We have the protoplasm of the liver cell remaining almost intact, just as does the protoplasm of the connective-tissue cell. It, along with the nucleus, which retains the greater part of its activity, is simply pushed aside, and on the absorption of the fat resumes all its previous activity. The liver cells, too, have the power of taking up pigment derived from the blood, and in normal conditions of breaking it up rapidly, and probably of excreting the products into the bile-ducts in the form of bile-pigment. Where, however, as in chronic venous congestion, their nutrition is interfered with we find not only breaking down of the liver cells due to the internal metabolism or the conversion of their own protoplasm into fat by the breaking down of its own proteid substance; in such cases we also find that pigment accumulates in these cells, pigment that is not readily got rid of, and which remains in them just as pigment remains in degenerating connective-tissue cells or leucocytes.

(To be concluded.)

\section{CHRONIC TRAUMATIC ARTHRITIS.}

BY W. ARBUTHNOT LANE, M.S ,

ASSISTANT SURGEON TO GUY'S HOSPITAL, AND TO THE HOSPITAL FOR SICK CHILDREN, GREAT ORMOND STREET.

Mr object in writing this paper is to make another attempt to separate from the several groups of conditions comprised by the terms "rheumatoid arthritis," "rheumatic gout," "osteo-arthritis," "arthritis deformans," \&c. one which is very definite in its history, clinical symptoms, and pathology. As far as I know, no author has recognised this class as a separate one, while pathologists do not pretend to make any such distinction. As its causation is entirely mechanical, I would suggest for it the term "tranmatic arthnitis," to which I would add "chronic," for the reason that the condition develops slowly, and when once produced it continues to progress during the lifetime of the patient. Some objection may be urged against the universal appropriateness of this term, since the condition to which I wish again to call attention does not always arise from the severe contusion of the joint as the result of a single injury; but it may, and frequently does, come about in consequence of the excessive use of a joint, or of any alteration in its normal anatomy and physiology, by means of which pres. sure is transmitted habitually from one bone to another in an abnormal manner; or it may result from a joint being called upon to sustain and transmit an amount of force greater than the particular human organism was evolved to support. In a paper in the Transactions of the Pathological Society, 1886, entitled the "Causation and Pathology of Rheumatoid Arthritis and of Senile Changes," I showed that the very large proportion of the pathological changes which are regarded as evidence of the so-called disease "rheumatoid arthritis" depend for their production upon factors which are simply mechanical. The careful clinical study of the conditions comprised under the term "rhen. matoid arthritis" \&c. by recent observers ${ }^{1}$ has resulted in the differentiation of two distinct groups of joint diseasesone, namely, rheumatoid arthritis, in whose causation rheumatism forms no part, and whose symptoms are very complicated and by no means limited to the changes in the joints, and the other rheumatic arthritis which results solely from rheumatism. To these two groups I would then add a third-chronic traumatic arthritis, - and I would urge that while it forms a not inconsiderable share of what is clinically regarded as rheumatoid arthritis \&s., it provides almoso solely all the conditions which in our museums are repre. sented as specimens of that disease.

I find that in the dead body the following changes are almost universally supposed to be characteristic of that vague condition rheumatoid arthritis, the term being here used in its older and more comprehensive acceptation, namely :-1. Removal of articular or inter-articular cartilage. 2. More or less destruction and eburnation of opposing surfaces of bone deprived of their articular cartilage. 3 . The formation of cartilage, or more usually of bone, upon the margins of the articular surfaces altering their form and increasing their area. These growths may occasionally extend in to the ligaments or less frequently develop primarily in them. To these changes the terms " osteo-phy tic growth," "lipping," \& e, are applied. 4. Hypertrophy of the synovial membrane so as to form more or less abundant masses or fringes consisting of branched pedunculated processes, which in some cases contain niuch fat, and in others smal masses of cartilage or bone. These occupy the varying in tervals between the altered articular surfaces, and often secrete an abnormally large quantity of synovial fluid. This may be so abundant as to distend the capsule and to cause much relaxation of ligaments. It is highly probable that these or similar changes may be found in patients who have been affected for many years by either of the groups of rheumatoid or rheumatic arthritis. They must come but rarely under the observation of the pathologist, and they are represented hardly if at all on the shelves of our museums.

I will now priceed to illustrate briefly the several conditions to which I would apply the term "chronic traumatio arthritis."

A man, past middle age, falls heavily upon his side, striking the great trochanter with much force. In consequence of this injury he experiences much local pain in the hip-joint, which is aggravated by movement of it. If the legs be measured they are found to be of equal lengtb. The patient finds that after the iajury his leg is not so reliable as it was before, that the movement of complete flexion of the hip-joint is accompanied with discomfort at first, and later with pain, so much so that any movement of the joint is avoided as much as possible. He also suffers discomfort or pain in the joint in damp weather, or during the cold east winds. I believe that it is the similarity of this pain to that felt by weumatic patients that has led clinical observers to include this traumatic condition among those classified under the o?d headings of "rheumatoid arthritis" \&c. I need hardly point out that after any injury to a bone, such as fracture \&c., the sufferer of ten complains of the same kind of pain under the same atmospheric conditions. After a time the patient notices that the damaged

Osteo-arthritis : Kent Spender. Rheumatic Diseases (so-called) Hugh Lane and Grifter 
leg has become shorter than its fellow, and actual measurement shows that this is the case. This shortening increases during the life-time of the patient if he continues to throw his weight upon the articulation. The joint becomes progressively less secure, until at length the weight of the body cannot be supported by it at all in many cases. Soon after the receipt of the injury grating can be felt on flexing or extending the joint, and this sensation becomes more marked as time goes on. If such a joint be examined after death, the head of the femur and the acebabulum are found to be completely altered in form and character. The opposing surfaces of the innominate bone and femur are rubbed down, sclerosed, and eburnated, while around these eburnated surfaces there is an abundant deposit of more or less dense bone, whose obvious function is to compensate for the altering direction in which force is transmitted through the joint and to render it as secure as possible under the circumstances. The capsule has become thin and lax, and has ceased to perform most of its normal functions. The synovial membrane is bulky in the joint and contains an abnormal amount of synovia. The causation of the several changes here described is quite obvious. The opposing surfaces of articular cartilage have been bruised sufficiently to interfere with their vitality, and there being no means of replacing the damaged tissue it is removed. The habitual transmission of force through the damaged joint results in the progressive destruction of the remainder of the articular cartilage, in the exposure, eburnation, and the progressive gradual destruction of the subjacent articular lamella of bone. These changes are followed by the others already described, the factors determining their evolution being solely mechanical. Is there any argument in favour of applying to this condition such a term as "rheumatoid arthritis" or any of its synonyms? A change, similar in cbaracter, though slighter than that just described, which develops to a varying degree in the hipjoint of labourers who are employed in carrying heavy loads, was misconstrued by anatomists, and led them to suppose that in old age the neck of the femur yielded, and that the head occupied a lower level than in the adult in consequence. In the paper already referred to I pointed out how this fallacy arose, and I have since been pleased to see that so keen an anatomist as Professor Humphry has in a recent paper denied that any such change takes place in the obliquity of the neck of the femur in old age.

The following serves as another instance of chronic traumatic arthritis. A man falls heavily on the outer surface of his shoulder. He suffers from much pain at the time, and he can neither bear the shoulder-joint to be moved nor can he perform any active movement himself, on account of the pain associated with it. If the shoulder be examined no fracture can be made out, and the outlines of the humerus and of the glenoid cavioy are normal. The symptoms progress as in the case of the hip. The shoulder becoming flattened, the patient is unable to abduct the humerus, and the movements of the shoulder joint become limited finally to a moderate amount of flexion and extension. On examining the joint after death the opposing surfaces of the head of the humerus and of the glenoid cavity are deprived of cartilage, the articular layer of bone is rubbed down and eburnated, the surface of the humerus is rendered flat, or but slightly convex, being much increased in breadth by the deposit of bone upon its margin. The articular surface of the scapula has undergone similar changes. The synovial membrane is bulky and the muscles of the shoulder, especially the abductor muecles, are much wasted. Here, again, the causation is quite obvious. Force is applied to the outer surface of the humerus in such a direction that its articular surface is driven forcibly against that of the scapula, and in a direction at right angles to the surface of the latter. Changes ensue in the manner already described in the hip.

I would instance still one more example in the same joint. A man receives a heavy blow or pitches heavily un the point of the shoulder, breaking his acromion pro. cess, whose loose fragment is driven forcibly downwards upon the upper surface of the humerus, bruising its articular surface severely, together with the intervening capsule and tendon of the supra-spinatus. Degenerative changes ensue in the contused portion of the articular cartilage, and gradually extend to more or less of the remainder of the cartilage of the head of tha humerus and to that of the glenoid cavity. The upper portion of the capsule, with more or less of the tendon of the supra-spinatus, is gradually destroyed, and the humerus comes sooner or later into direct contact with the movable fragment of the acromion. The constant movement of these bones upon one another results in further changes in both, the acromial portion becoming excavated, eburnated, and lipped. Theintracapsular portion of the biceps tendons is destroyed, the long head acquiring an attachment to the altered great tu berosity. Obher changes develop at the same time in the synovial membrame. I would call particular attention to this last injury, as it bears in an important manner on the elucidation of the causation and pathology of chronic traumatic arthritis. I showed ${ }^{2}$ some time ago that fracture of the acromion is of very common occurrence, and that the sup. posed rarity of the fracture depended upon a want of skill in recognising its existence. Since that paper I have been able to verify this statement most fully, both in the accident department, where the recent fractures were observed, and in the out-patient department, where the condition of non union which almost always results was seen. This non union is due to the fact that unless the accident is diagnosed, which it very rarely is, or unless it be accompanied by some more severe injury, as fracture of the upper end of the humerus, the shoulder is not kept at rest for a sufficient time to allow of the fracture of the acromion uniting by bone, and as a consequence a false joint forms. When the blow which produced the fracture was sufficiently severe to contuse the articular cartilage of the head of the humerus and the intervening soft parts, the changes $I$ have just described follow.

Anatomists are largely responsible for the non-recognition of these fractures, since they have chosen to regard the resulting ununited conditions as examples of non-nnion of an epiphysis, and, so far as I can see, upon no reliable evidence whatever. Some surgeons have readily accepted the statements of the anatomists apparently without investigating them. They bave, however, observed the frequent association of this so-called ununited epiphysis with the presence of so-called rheumatoid changes in the shoulder-joint, and have explained the cause of thei association in various ways. One has gone so far as to assert that in cases of chronic rheumatoid arthritis of the shoulder-joint a disunion of the acromial epiphysis from the rest of the bone takes place. Such a statement hardly calls for criticism, and I only mention it to indicate the frequency with which those changes which the pathologist regards as characteristic of the disease, rheumatoid arthritis of the shoulder-joint, are found associated with ununited fracture of the acromion. Can anything be more obvious and simple than the mode of development of an ununited fracture of this process of bone, if one considers for one moment its position and muscular attachments, together with the difficulty of retaining the broken fragment at rest for any length of time, even if the fracture be recognised? The only two examples of bony union which I have observed followed a fracture of the acromion, which was accompanied by a severe comminuted fracture of the apper end of the humerus. $O$ wing to the pain resulting from the presence of the latter fracture the shoulder was kept at rest sufficiently to allow of bony union. Though I might multiply such instances indefinitely, I think I have said enough to show that a severe injury to a joint is liable to be followed by the development of such pathological conditions as we are accustomed to regard as evidence of the presence of rheumatoid arthritis.

I will now allude but very briefly to the remarkable changes which develop in the joints of men or beasts of burden accustomed to follow some laborious occupation for many years, since I have described them very fally in preceding papers. They were all regarded as examples of rheumatoid arthritis on the post-mortem table, and also not uncommonly during lifetime. They consist in the removal of articular cartilage, the sclerosing, eburnation of the exposed bones, the alteration in the forms of the articular surfaces-sometimes by considerable excavation of bone, sometimes by the abundant deposit of bone upon the margins of the articular surfaces, - by the formation of masses of bone upon the floor of cavities-such as the olecranon and coronoid depressions, - the removal of fibro-cartilage frcm the sacro-iliac and vertebral joints, the formation of large prominences of bonelocking these joints, the fusion of opposirig surfaces of bone, \&c. Again, precisely similar changes take

The Pathology of Changes producex by Pressure in the Bony Skeleton of the Trunk and Shoulder Girdle: riny's Hospital Report, 
place in joints if the direction of force transmitted through them varies from the normal and the articulation is made to perform a function for which it was not originally fitted. I refer to changes occurring in the joints in knock.knee, bow-legs, lateral curvature, in cases of shortening of one leg by fracture or disease, \&c. I am well aware that both rheumatoid and rheumatic arthritis, and especially the Former, are very prone to affect a joint damaged in its anatomical detail, and consequently in its physiology, by occupation or injury, and this has been pointed out very slearly by Dr. Hensley of Bath, and it may in some few cases be difficult to state whether the condition is one whose causation is solely traumatic, or whether added to this we have in addition a factor whose mode of action is much more complex; yet I would assert that in the very large majority of instances of the condition which $I$ have called chronic traumatic arthritis there is no difficulty in recognising it either clinically or in the dead body, and that therefore we should no longer include this definite group under a series of names which only serve as a cloak to ignorance, but give it a definite and explanatory title.

St. Thomas's-street, S.E.

\section{DBSERVATIONS ON CERTAIN DISPUTED POINTS IN RENAL PATHOLOGY. ${ }^{1}$}

$$
\text { Bx A. G. AULD, M.D. }
$$

FIRST I shall describe what seems to be the true interpretation of the morbid changes found in the pia mater and in the skin, then certain lesions in the Malpighian bodies, and lastly I shall deal with some considerations based on anatomical data respecting the cause of the polyuria which is so constantly observed in the contracting form of Bright's kidney. I have been enabled, through the courtesy of Dr. Coats, to examine the pia mater of all brains examined in the Western Infirmary during a period of fifteen months. In each case the membrane was carefully hardened in Müller's fluid and alcohol, then dehydrated, and soaked for some days in Hamilton's freezing fluid A. From the resulbing sections some valuable facts were obtained; and, in particular, I bave been enabled to make out by means of high powers what seems to be the true nature of the hyalinefibroid thickening of Gull and Sutton. As is well known, these anthors, in their celebrated papers, contended that in Bright's disease the bloodvessels throughout the body, and especially those of the pia mater, had their outer coats thickened by the deposition therein of a byaline fibroid material, probably derived from the blood. The muscular coat in such vessels they conceived to be atrophied, while the inner coat frequently, but by no means invariably, partook of hyperplastic change. This theory received a most lamaging criticism from Johnson, who strongly maintained the traly hy pertrophic condition of the muscular layer, while the so-called "hyaline fibroid" he alleged to be a delusive result of reagents, such as glycerine and acidulated mounting huids. In 1873 Grainger Swewart pointed out that, while mounting media undoubtedly had some influence in producing the hyaline appearance, not a few cases exhibited a true fibrous thickening. Dickinson subsequently lent his adherence to this view, but the precise nature of the change is atill the theme of debate. According to my observations, it may be stated generally that in Bright's disease all the costs are thickened. In the vast majority of cases the inner coat shows endarteritis. This change seems very constant in fatty kidneys in adults, though it is occasionally absent in contracting kidney. As regards the muscular coat, there can be no room for doubt that Johnson's contention is the correct one. Further, it was maintained by this eminent observer that a longitudinal layer of muscle-cells could be observed alongside the hy pertrophied circular layer in some of these cases. It was asserted by Dr. Bryan Waller that this was an impossibility, and that Dr. Johnson mistouk the cellular strata of the thickened inner coat for a layer of muscle cells. I think, however, that on looking at one of the specimens we will be savistied that to the inside of the hypertrophied circular coat longitudinal muscular fibres

A paper delivered, with microscopic demonstrations, before the Glasgow Pathological and Clinical Society, Jan. 11th, 1892. exist which can in no way be confounded with the proximate layers of the thickened intima. There are, however, some cases in which the muscular hypertrophy is far from being a marked feature, and when the disease is far advanced degenerative changes of an aggressive character attack the hypertrophied fibres. In the case of the outer coat it is plainly shown from these preparations that it is thickened, and the question is, What is the nature of this thickening? Now, after eliminating the possibility of amyloid disease by careful staining, I have invariably made out by means of very high powers the existence of branched corpuscles, with dividing nuclei, either embedded in or lying alongside the hyaline-fibroid formation. It is clear that this formation owes its origin to a slowly progressing inflammatory new formation of connective tissue, and that the effect of reagents is, after all, but unimportant. Not only so, but this corpuscular activity is manifest throughout the connective tissue of the pia mater generally, leading to a more or less general thickening.

As regards the skin, the sections show that the muscular coats of the arterioles are much thickened. This is gentrally to be observed. In one case, in which the cedena was a very marked feature, the derma is unduly cellular; clusters of leucocytes seem to be scattered throughout its substarce. It seems as though some inflammatory process were at work, and hence we find here considerable support to the view of Cohnheim as to the cause of this form of odema.

With regard to the lesions of the kidney itself in chronic Bright's disease, examination of a large number of kidneys has led me to recognise two great divisions. In one, the lesions of the glomeruli and of Bowman's capsule are very marked, and overshadow the other lesions; in the other class the reverse holds good. ${ }^{2}$ Greenfield recognises the lesions of the capsule as pericapsulitis or cellular infiltration and tissue-formation occurring chiefly outside this structure, as hyaline thickening of the capsule itself, and as endo-capsulitis or the formation of concentric laminæ of cells and tissue inside the capsule between it and the glomerular tuft. Greenfield regards the cells lining the capsule to be capable of originating this formation. Now, in acute glomerulitis it is undoubted that layers of epithelial cells are formed inside the cap:ule, derived from germination of its lining epithelium. But in chronic glomerulitis this is no longer seen. lustead we have the following changes:-1. The epithelial cells covering the glomeruli swell and assume a peg-top or columnar shape (well seen in the section placed under the microscope); they then fall into the periglomerular space, and assume a rounded form. 2. A similar desquamation of the cells lining the capsule occurs, but without the intervention of the columnar shape. 3. The connective tissue nuclei, and likewise the nuclei of the capillary walls of the Malpighian tufts, germinate, multiply, and give rise to a new growth of tissue between the capsule and the tuft, the capillaries undergoing hyaline thickening the while. In one specimen we see a band of new tissue stretching across the peri-glomerular space to which numerous epithelial cells have attached themselves - a phenomenon which has been questioned by Ziegler and some others. 4. Not infrequently the capsule itself becomes slit up into several layers, giving the appearance of two or more capsules for part of its circumference. This dissecting process is accompliehed by cells from the surrounding connective tissue. In other and more advanced cases the capsule appears striated and concentrically thickened, and blends with the sclerosed glomerular tuft. It is difficult to see why these lesions should occur in some cases and not in others; they are by no means confined to scarlatinal cases, and, moreover, in post-scarlatinal nephritis which has aseumed a chronic form they are occasionally absent.

Lastly, I propose to advance a few original considerations respecting the cause of the polyuria which is such a cha. racteristic symptom of contracting kidney-considerations based, I may say, almost entirely on the histological appearances which this kidney presents. It is the universal custom to ascribe the excess of urine to one or other, or else a com. bination, of the following factors. Increase of the blood pressure geverally ; increase of the blood pressure locally in the glomeruli, arising from obliteration of capillary areas; inability of the tubular epithelium to absorb the water passing from the glomeruli ; and increased permeability of

2 I purposely omit here particular reference to changes in the parenchyma. 